\title{
Effect of intravitreal conbercept injection on VEGF-A and -B levels in the aqueous and vitreous humor of patients with proliferative diabetic retinopathy
}

\author{
YUNDA ZHANG $^{1}$, ZHAO GAO $^{1,2}$, XIMEI ZHANG $^{1}$, ZHIGANG YUAN $^{1}$, \\ TAO MA ${ }^{1}$, GAIYUN LI ${ }^{1}$ and XIAOHONG GAO ${ }^{1}$ \\ ${ }^{1}$ Department of Vitreoretinopathy, Shanxi Eye Hospital, Taiyuan, Shanxi 030002; \\ ${ }^{2}$ Department of Ophthalmology, Tianjin Medical University General Hospital, Tianjin 300052, P.R. China
}

Received July 20, 2020; Accepted January 6, 2021

DOI: $10.3892 /$ etm.2021.9763

\begin{abstract}
The present study aimed to investigate the mechanisms of anti-VEGF treatment prior to eye surgery to reduce intraoperative bleeding. A total of 30 patients with proliferative vitreoretinopathy after clinical diagnosis were enrolled in the present study as the surgical group. Furthermore, 30 patients underwent intravitreal injection of the anti-VEGF drug conbercept and were considered the drug pretreatment group. The aqueous and vitreous humors from the eyes of patients in the surgical group were drawn during pars plana vitrectomy surgery. The aqueous humor in the eyes of patients in the drug pretreatment group was drawn prior to conbercept treatment and seven days after conbercept treatment immediately prior to surgery. The vitreous humor in this group was only taken during surgery. Furthermore, ELISA was used to detect the levels of VEGF-A and -B in the aqueous and vitreous humors. Semi-quantitative determination of VEGF-A and VEGF-B levels in fibrovascular proliferative membranes was performed using immunohistochemical staining. The results indicated that in the drug group, the levels of VEGF-A in the aqueous humor of patients prior to and after conbercept injection were $197.66 \pm 48.00$ and $3.39 \pm 2.54 \mathrm{pg} / \mathrm{ml}$, respectively. The levels of VEGF-A in the vitreous humor of patients in the surgical and drug groups were $267.53 \pm 179.60$ and $21.43 \pm 5.81 \mathrm{pg} / \mathrm{ml}$ after injection, respectively. The levels of VEGF-B in the aqueous humor of patients prior to and after conbercept injection were $13.66 \pm 3.30$ (before injection) and $2.17 \pm 0.94 \mathrm{pg} / \mathrm{ml}$ (after injection), respectively. The levels of VEGF-B in the vitreous humor of patients in the surgical and drug groups were $127.36 \pm 16.72$ and $18.56 \pm 9.82 \mathrm{pg} / \mathrm{ml}$ after injection, respectively $(\mathrm{P}<0.05)$. Furthermore, in the drug
\end{abstract}

Correspondence to: Dr Xiaohong Gao, Department of Vitreoretinopathy, Shanxi Eye Hospital, 100 Fudong Street, Xinghualing, Taiyuan, Shanxi 030002, P.R. China

E-mail: violetdaisynn@126.com

Key words: conbercept, VEGF, humor, diabetic retinopathy group, the surgery time, bleeding and capillary formation were significantly reduced compared with those in the surgical group. Taken together, these results suggested that the levels of VEGF-A and -B decreased significantly in the aqueous humor of patients with PDR after conbercept injection. Furthermore, the levels of VEGF-A and -B in the vitreous humor of patients dropped significantly in the drug group compared with those in the surgical group. These results provide theoretical clinical support for the preoperative application of conbercept for patients with PDR.

\section{Introduction}

Diabetic retinopathy (DR) is a microvascular complication of diabetes mellitus (DM) and remains the most common cause of vision loss worldwide (1). It is estimated that an increasing number of individuals will suffer from DR and its complications in the future. Almost all patients with Type I and $60 \%$ of patients with Type II diabetes have developed a certain degree of retinopathy after 20 years (2). Proliferative DR (PDR) is a type of DR that severely threatens vision and features vitreous hemorrhage and neovascularization of the eye fundus and iris (3). In China, the major method of treating PDR is laser photocoagulation, but this technique may damage retinal function, which makes it an imperfect strategy. Usually, pars plana vitrectomy (PPV) is used to cure patients with PDR and is a useful tool that avoids different kinds of advanced complications. However, during or after PPV, vitreous hemorrhage may lead to severe consequences including retinal detachment (4).

With deeper recognition of the pathologic mechanisms of DR, medical therapy has made significant progress, including the use of glucocorticoids, anti-vascular endothelial growth factor (VEGF) drugs, anti-platelet-derived growth factor drugs, nonsteroidal anti-inflammatory drugs and drugs that improve the function of the optic nerve. Anti-VEGF agents have gained popularity in the treatment of diabetic macular edema. VEGF destroys the tight junction between retinal vascular endothelial cells and leads to the destruction of the blood-retinal barrier, leading to intravascular fluid leakage and macular edema (5). Ostensibly, VEGF is recognized as a crucial mediator in diabetic macular edema and vitreous injection of 
anti-VEGF agents has become a promising therapy for PDR. Intravitreal injection of VEGF inhibitors reduces the leakage of diabetic neovascular lesions and is suitable for vitreous hemorrhage $(6,7)$; it improves the visual acuity and prognosis of patients with PDR and reduces surgical complications in patients with PDR. Furthermore, intravitreal injection of VEGF inhibitors is less likely to cause cataracts and high intraocular pressure than vitreous injection of glucocorticoids (5). In addition, the vision prognosis of patients receiving continuous injection of VEGF inhibitors within two years is better than that of patients treated by panretinal photocoagulation (8).

In PDR-associated retinal hypoxia, leukocytes adhere to retinal capillaries under hyperglycemic conditions, leading to augmentation of vascular permeability and capillary occlusion. As a consequence, VEGF is stimulated to induce retinal ischemia, which is closely correlated with neovascularization, vitreous hemorrhage and severe vision loss $(9,10)$. Therefore, angiogenesis is closely related to the occurrence and development of PDR, and VEGF has a pivotal role in PDR by promoting angiogenesis and vascular hyperpermeability. It is well known that VEGF-A activates both VEGF receptor (VEGFR)-1 and VEGFR-2, while VEGF-B binds selectively to VEGFR-1 (11). The VEGF-A protein targets endothelial cells and has multiple effects, including cell growth and migration, angiogenesis and increased vascular permeability (12). The function of VEGF-B in ophthalmology remains controversial, although it has been investigated in cardiovascular disease, diabetes and cancers (13). In the present study, changes in the concentration of VEGF-A and VEGF-B after intravitreal injection of conbercept were investigated.

Conbercept is a soluble VEGFR protein and is composed of the extracellular domain 2 of VEGFR-1 and extracellular domains 3 and 4 of VEGFR-2. It has been suggested that conbercept pretreatment accelerates postoperative vitreous clear-up and maintains stable visual acuity restoration in patients with PDR (11). However, changes in VEGF-A and VEGF-B levels in the aqueous and vitreous humors of patients with PDR after intravitreal injection of conbercept have been insufficiently reported in previous studies and require to be determined. In the present study, whether injection of a VEGF inhibitor would decrease VEGF levels was assessed and the efficacy of conbercept for the treatment of PDR was also evaluated.

\section{Patients and methods}

Patients. A total of 60 patients diagnosed with diabetic retinopathy induced by type 2 diabetes at Shanxi Eye Hospital (Taiyuan, China) between January 2016 and January 2018 were examined. The cohort included 34 males and 26 females with an age of 32-76 years, a mean age of $55.67 \pm 8.21$ and a mean proportion of glycosylated hemoglobin of $7.45 \pm 1.23 \%$. All of the samples were monocular and patients had been treated by surgery first. All patients underwent fundus photography, mydriatic indirect ophthalmoscopy and B-ultrasound, and certain patients underwent fundus fluorescein angiography. The examination results were in accordance with the PDR staging criteria of the fundus disease group of the Chinese Ophthalmological Society from 1985 (11) and patients in phase V and VI were included. Patients with other ocular diseases, including nondiabetic retinopathy of retinal arteriovenous obstruction, high myopia and polypoid choroidal neovascularization, were excluded. Furthermore, patients who underwent anti-VEGF therapy in the past 12 months and vitrectomy, patients with severe organic diseases (such as severe heart disease, liver and kidney failure and cancer), patients during a menstrual period or who were taking aspirin or other anticoagulants were also excluded. The grading for preoperative vitreous hemorrhage was performed as in a previous study (14). Grade 0 , no vitreous hemorrhage; grade 1 , visible fundus details, but the retinal nerve fiber layer or small blood vessels cannot be assessed; grade 2, more bleeding and large blood vessels of the optic disc compared to grade 1; grade 3 , only a slight reflex of the fundus and only the disc is visible; grade 4 , no red light reaction and the fundus cannot be seen. This study was approved by the research ethics board of Shanxi Eye Hospital (Taiyuan, China; no. 2019B01). This study was a retrospective analysis summarizing our previous cases and analyzing their data.

Interventions. Patients were divided into the surgical group and the preoperative intravitreal conbercept group (the drug group). Patients in the surgical group received PPV directly and their aqueous and vitreous humors were extracted during PPV surgery. The aqueous humor of patients in the preoperative intravitreal conbercept group was extracted prior to treatment and seven days after intravitreal conbercept injection before PPV. The vitreous humor of patients in the preoperative intravitreal conbercept group was extracted during PPV surgery. All of the patients received an explanation of the off-label use of conbercept in PDR and its potential benefits and risks. Prophylactic topical antibiotics (levofloxacin; Selleck Chemicals) were instilled for 3 days prior to treatment. After topical anesthesia and sterilizing the operating field, $0.5 \mathrm{mg}$ (0.05 ml) conbercept (Chengdu Kanghong Biotechnologies Co., Ltd.) was injected intravitreally at the superior temporal pars plana (4 $\mathrm{mm}$ posterior to the limbus). After the injection, the intraocular pressure was measured and prophylactic topical antibiotic drops were instilled for another 3 days. Standard 25-gauge three-port PPV was performed one week after the intravitreal injection by the same experienced ocular surgeon. The intraoperative bleeding grades were assigned as follows (15): Grade 0, no bleeding or small bleeding spots with no requirement for hemostasis; grade 1, self-stopping small bleeding or bleeding that may be stopped through elevated perfusion pressure or glass-cutting head compression; grade 2, electrocoagulation is required to stop the bleeding due to the high bleeding volume; grade 3 , the blood clot formed exceeds the posterior pole or affects the surgical field of view.

Aqueous humor extraction. A 1-ml syringe was used to enter the anterior chamber at the edge of the horn-sclera. The fluid was extracted from the anterior chamber and rapidly filled in an Eppendorf tube, followed by centrifugation in a low-temperature ultracentrifuge at $2,000 \mathrm{x} \mathrm{g}$ for $20 \mathrm{~min}$ at $4^{\circ} \mathrm{C}$. The supernatant was obtained and stored in the freezer at $-80^{\circ} \mathrm{C}$. Aqueous humor was collected via anterior paracentesis prior to conbercept injection and at the beginning of vitrectomy in the operation room. The aqueous humor sample was collected in a tuberculin syringe, filled in a sterile Eppendorf tube and stored at $-80^{\circ} \mathrm{C}$ prior to use. 
Table I. Preoperative vitreous hemorrhage and adhesion grades between the neovascular proliferation membrane and retina in patients with proliferative diabetic retinopathy.

\begin{tabular}{|c|c|c|c|c|c|c|c|c|}
\hline \multirow[b]{2}{*}{ Group } & \multirow[b]{2}{*}{ Eyes $(n)$} & \multicolumn{4}{|c|}{ Preoperative vitreous hemorrhage grade (n) } & \multicolumn{3}{|c|}{ Retinal adhesion grade $(\mathrm{n})$} \\
\hline & & 1 & 2 & 3 & 4 & 1 & 2 & 3 \\
\hline Drug & 30 & 2 & 11 & 14 & 3 & 14 & 18 & 8 \\
\hline Surgery & 30 & 4 & 13 & 11 & 2 & 11 & 23 & 6 \\
\hline $\mathrm{U}$ & & \multicolumn{4}{|c|}{-0.075} & \multicolumn{3}{|c|}{-0.232} \\
\hline $\mathrm{P}$-value & & \multicolumn{4}{|c|}{0.940} & \multicolumn{3}{|c|}{0.816} \\
\hline
\end{tabular}

Groups were compared with the Mann-Whitney U-test.

Vitreous humor extraction. A $25 \mathrm{G}$ vitrectomy head was used to cut $0.5 \mathrm{ml}$ vitreous humor in the center of the vitreous cavity, and then the vitreous humor was quickly added to an Eppendorf tube. The sample was placed into a low-temperature ultracentrifuge, centrifuged at $2,000 \mathrm{x}$ g for $20 \mathrm{~min}$ at $4^{\circ} \mathrm{C}$ and stored in a $-80^{\circ} \mathrm{C}$ freezer.

Proliferative membrane extraction and histopathological examination. The neovascular proliferative membrane of patients with PDR was removed during the operation and its size was $\sim 2.0 \times 2.0 \times 0.5 \mathrm{~mm}^{3}$. The membrane was fixed in a $10 \%$ formalin solution and then stored in a wax block. The paraffin specimen was cut into $3-\mu \mathrm{m}$ slices. The number of blood vessels in the fibrovascular proliferative membrane was determined by H\&E staining. Paraffin sections were dewaxed, hydrated, stained with hematoxylin, acidified with hydrochloric acid and observed under a light microscope (magnification, $\mathrm{x} 200$ ). Five fields were observed for each specimen to obtain the average number of blood vessels.

Semiquantitative determination of VEGF-A and VEGF-B in fibrovascular proliferative membranes by immunohistochemical staining. Fibrovascular proliferative membrane sections were deparaffinized in water and washed with PBS three times for $10 \mathrm{~min}$. Subsequent to hot antigen retrieval (citrate buffer, $\mathrm{pH} 6.0,100^{\circ} \mathrm{C}$ for $25 \mathrm{~min}$ ), $5 \%$ bovine serum albumin (Beijing Solarbio Science \& Technology Co., Ltd.) blocking solution was added, followed by incubation at room temperature for $20 \mathrm{~min}$. The sample was then incubated with primary antibody (anti-VEGF-A, cat. no. ab51745; anti-VEGF-B, cat. no. ab185696; 1:200 dilution, Abcam) at $4^{\circ} \mathrm{C}$ overnight, followed by incubation with the secondary antibody (cat. no. ab205718, 1:5,000 dilution, Abcam) at $37^{\circ} \mathrm{C}$ for $20 \mathrm{~min}$. After washing with PBS three times, the sample was incubated with strept avidin-biotin complex (Beijing Solarbio Science \& Technology Co., Ltd.) at $37^{\circ} \mathrm{C}$ for $20 \mathrm{~min}$. Diaminobenzidine staining was then performed prior to washing three times with distilled water. Hematoxylin stain was applied for 2 min followed by washing with water three times, acidification for $2 \mathrm{sec}$, washing three times, dehydration and sealing with neutral gum. For the negative control, PBS was used instead of the primary antibody. Images of the immunohistochemically stained tissue sections were obtained under a light microscope using Image-Pro Plus software (version 6.0;
Media Cybernetics, Inc.) (16,17). The images were analyzed with this software and the average optical density (AOD) value of the positive region was calculated. A total of three regions were randomly selected for each specimen and the average AOD value was calculated.

ELISA. The concentrations of VEGF-A and VEGF-B in aqueous and vitreous humor samples were determined by using an ELISA kit (VEGF-A kit, cat. no. F3255; VEGF-B kit, cat. no. F3254; Shanghai Westang Bio-Tech Co.,Ltd.) according to the manufacturer's protocol. The plates were incubated with capture antibodies and were then covered with $100 \mu \mathrm{l}$ standard, controls or samples for $40 \mathrm{~min}$ at room temperature. Subsequently, the plates were washed and incubated with the detection antibody for $20 \mathrm{~min}$ at room temperature. The plates were washed again and the substrate was added. The reaction was stopped after 15 min of incubation at room temperature in the dark, the stop buffer was added and the plates were read at $450 \mathrm{~nm}$.

Statistical analysis. SPSS 20.0 software (IBM Corp.) was applied for data analysis. Measurement data were expressed as the mean \pm standard deviation. Student's t-test was used to compare the mean difference of numerical variables between groups, the Chi-square test was used for analyzing count data, and the Mann-Whitney U-test was performed for non-parametric data. $\mathrm{P}<0.05$ was considered to indicate a statistically significant difference.

\section{Results}

Preoperative vitreous hemorrhage and vitreoretinal adhesion are similar in the surgery group and the drug group. The study included 34 males and 26 females with an age range of 32-76 years, a mean age of $55.67 \pm 8.21$ and a mean proportion of glycosylated hemoglobin of $7.45 \pm 1.23 \%$. According to a previous study, the classification criteria for vitreoretinal adhesion are as follows (18): Grade 0, no adhesion; grade 1, $<3$ point-level adhesions; grade 2, >1 extensive adhesion or adhesion at the optic disc, macula lutea or vascular arch; grade 3, vitreoretinal adhesion beyond the peripheral part. As presented in Table I, the P-values for the grades of preoperative vitreous hemorrhage and retinal adhesion between the surgery and drug groups were 0.940 and 0.816 , respectively. The 
Table II. Comparison of surgery time and intraoperative bleeding between the drug group and the surgery group.

\begin{tabular}{|c|c|c|c|c|}
\hline Item & Drug group $(n=30)$ & Surgery group $(n=30)$ & $\mathrm{t} / \chi^{2}$ & P-value \\
\hline Surgery time (min) & $54.54 \pm 10.93$ & $64.97 \pm 20.01$ & 2.51 & 0.01 \\
\hline Intraoperative bleeding grade & & & 6.37 & 0.04 \\
\hline 0 & 18 & 9 & & \\
\hline 1 & 12 & 20 & & \\
\hline 2 & 0 & 1 & & \\
\hline
\end{tabular}

The $\chi^{2}$ test was used for comparison of intraoperative bleeding.

Table III. Number of blood vessels in the proliferative membrane.

\begin{tabular}{lccc}
\hline Item & Surgery group $(\mathrm{n}=30)$ & Drug group $(\mathrm{n}=30)$ & $\mathrm{t}$ value \\
\hline Number of vessels & $32.17 \pm 5.80$ & $17.83 \pm 3.90$ & 11.23 \\
\hline
\end{tabular}

Values are expressed as the mean \pm standard deviation.
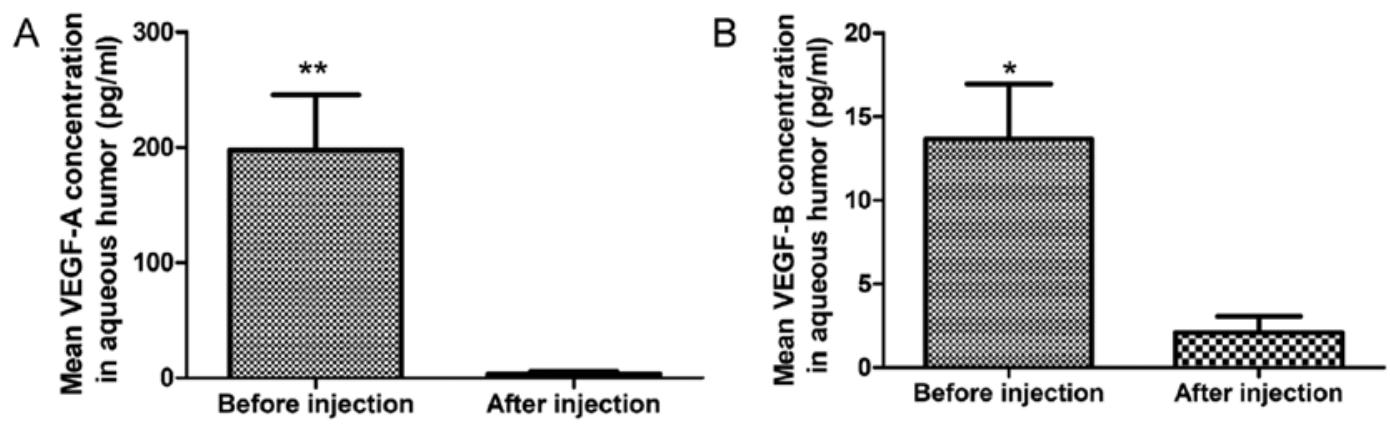

Figure 1. Comparison of (A) VEGF-A and (B) VEGF-B levels in aqueous humor prior to and after conbercept treatment in the drug group (P=0.001 and $\mathrm{P}=0.022$, respectively). ${ }^{* *} \mathrm{P}<0.01$ vs. after injection; ${ }^{*} \mathrm{P}<0.05$ vs. after injection. The drug group also received surgery.

P-values were both $>0.05$, indicating that the changes between the two groups were not significant. There were no significant changes observed in preoperative vitreous hemorrhage and vitreoretinal adhesion between the surgery and drug groups.

Reduced intraoperative bleeding in the drug group compared with that in the surgery group. As presented in Table II, the surgery time in the drug group was shorter than that in the surgery group and the difference was statistically significant $(\mathrm{P}=0.01)$. Intraoperative bleeding in the surgery group was more severe than that in the drug group and the difference was statistically significant $(\mathrm{P}=0.04)$.

VEGF-A and VEGF-B levels in aqueous humor prior to and after conbercept treatment in the drug group. As presented in Fig. 1, the mean VEGF-A concentration in aqueous humor was $197.66 \pm 48.00 \mathrm{pg} / \mathrm{ml}$ prior to conbercept treatment, which was higher than the concentration after conbercept treatment $(3.39 \pm 2.54 \mathrm{pg} / \mathrm{ml})$ and the difference was statistically significant $(\mathrm{P}=0.001)$. The mean VEGF-B concentration in aqueous humor prior to conbercept treatment $(13.66 \pm 3.30 \mathrm{pg} / \mathrm{ml})$ was higher than that after conbercept treatment $(2.17 \pm 0.94 \mathrm{pg} / \mathrm{ml})$ and the difference was statistically significant $(\mathrm{P}=0.022)$.

Comparison of VEGF-A and VEGF-B levels in aqueous humor between the surgical group and the preoperative intravitreal conbercept group. As presented in Fig. 2, the mean VEGF-A concentration in the aqueous humor of patients in the surgical group $(402.18 \pm 19.37 \mathrm{pg} / \mathrm{ml})$ was higher than that in the drug group $(3.39 \pm 2.54 \mathrm{pg} / \mathrm{ml})$ and the difference was statistically significant $(\mathrm{P}=0.001)$. Furthermore, the mean VEGF-B concentration in the aqueous humor of patients in the drug group was $2.17 \pm 0.94 \mathrm{pg} / \mathrm{ml}$, which was lower than that of patients in the surgical group $(27.77 \pm 2.52 \mathrm{pg} / \mathrm{ml})$ and the difference was statistically significant $(\mathrm{P}=0.001)$.

Comparison of VEGF-A levels and VEGF-B levels in vitreous humor between the surgical group and the preoperative intravitreal conbercept group. As indicated in Fig. 3, the mean VEGF-A concentration in the vitreous humor of patients in the surgical group $(267.53 \pm 179.60 \mathrm{pg} / \mathrm{ml})$ was higher compared with that of patients in the drug group $(21.43 \pm 5.81 \mathrm{pg} / \mathrm{ml})$ and 

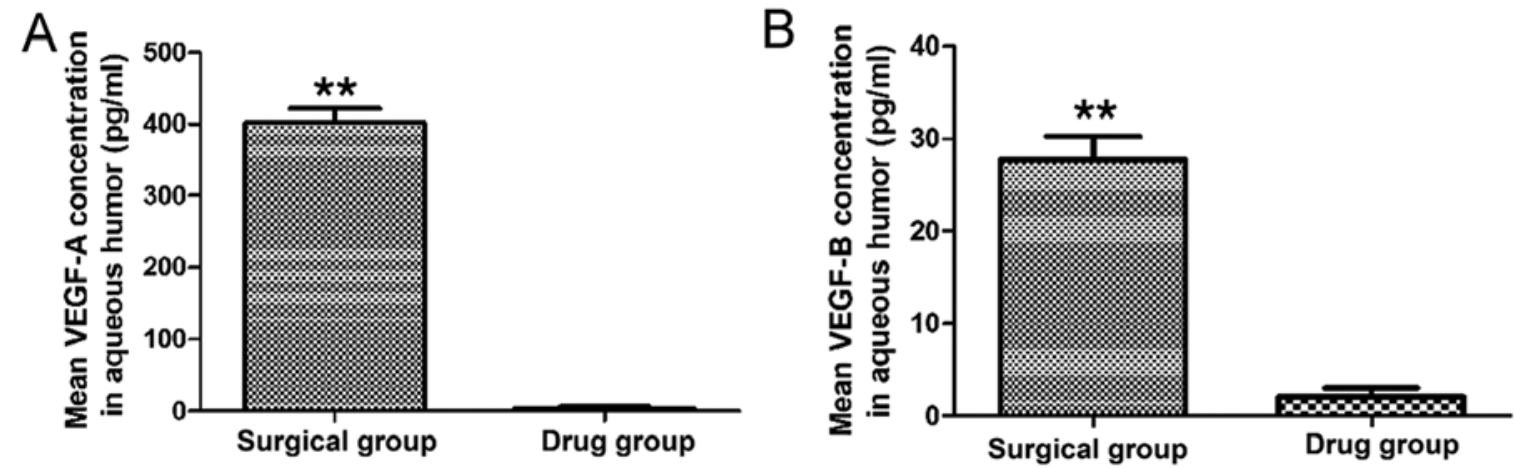

Figure 2. Comparison of (A) VEGF-A and (B) VEGF-B levels in aqueous humor between the surgical group and the drug group ( $\mathrm{P}=0.001$ for each). ${ }^{* * *} \mathrm{P}<0.05$ vs. drug group. The drug group also received surgery. In the operation group, aqueous humor was extracted during operation. In the drug group, the aqueous humor was extracted 7 days after the injection of conbercept and just before the operation.
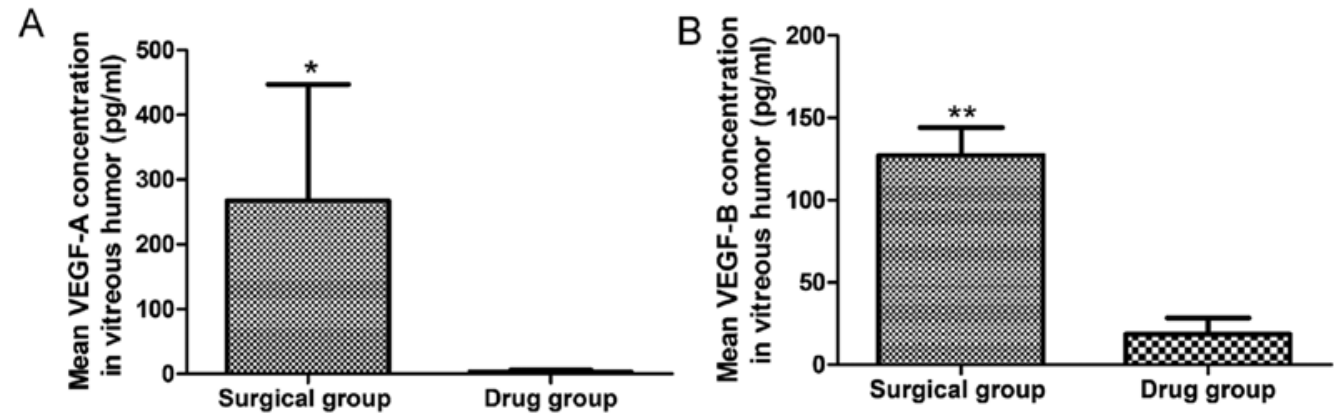

Figure 3. Comparison of (A) VEGF-A and (B) VEGF-B levels in vitreous humor between the surgical group and the drug group ( $\mathrm{P}=0.037$ and $\mathrm{P}=0.001$ ). ${ }^{*} \mathrm{P}<0.05$ vs. drug group. ${ }^{* *} \mathrm{P}<0.01$ vs. drug group. The drug group also received surgery. In the operation group, the vitreous humor was extracted during operation. The patients in the drug group received the operation 7 days after the injection of conbercept, and the vitreous humor was extracted during the operation.
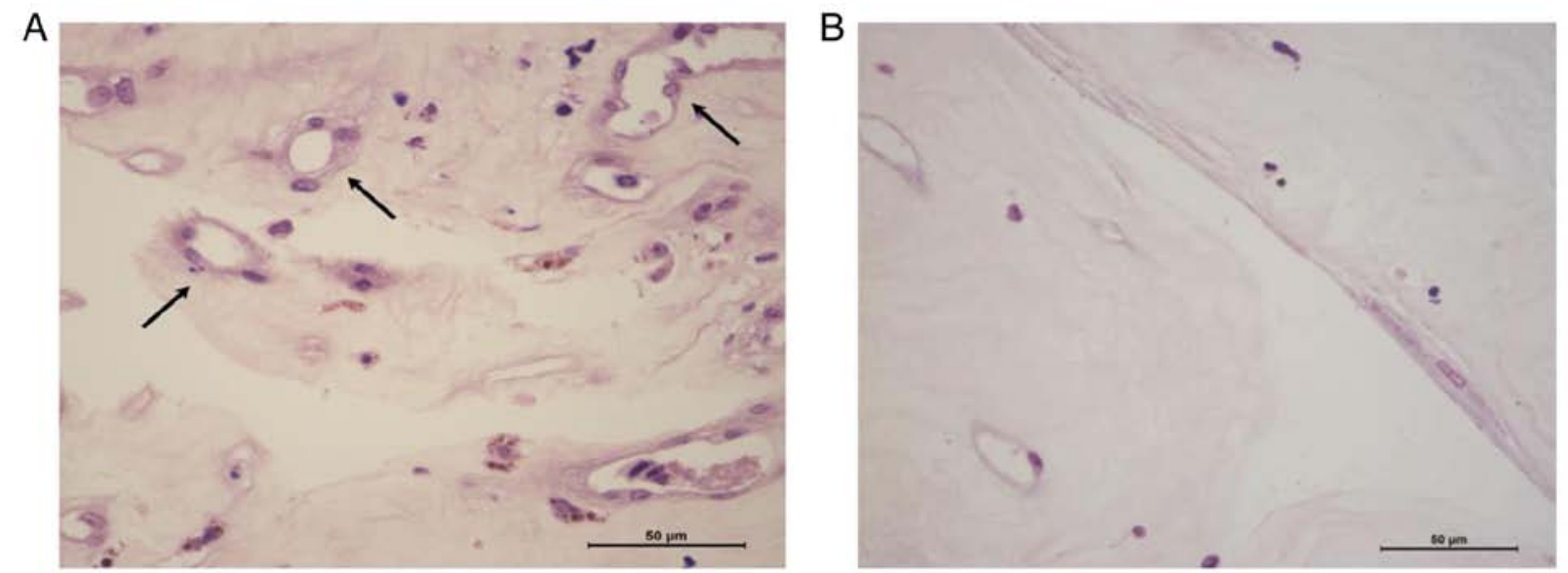

Figure 4. H\&E staining of the retinal proliferative membrane. (A) In the surgery group, the number of blood vessels was increased compared with the drug pretreatment group. (B) In the drug pretreatment group, the number of blood vessels was reduced compared with that in the surgery group. The arrows indicate blood vessels (scale bars, $50 \mu \mathrm{m}$ ). The drug group also received surgery.

the difference was statistically significant $(\mathrm{P}=0.037)$. Similarly, the mean VEGF-B level was higher in the vitreous humor of patients in the surgical group $(127.36 \pm 16.72 \mathrm{pg} / \mathrm{ml})$ compared with that of patients in the drug group $(18.56 \pm 9.82 \mathrm{pg} / \mathrm{ml})$ and the difference was statistically significant $(\mathrm{P}=0.001)$.

Reduced number of blood vessels in the proliferative membrane in the drug vs. surgery group. As presented in Fig. 4 and Table III, the number of blood vessels in the proliferative membrane in the drug treatment group was $17.83 \pm 3.90$, which was significantly lower than that in the simple surgery group $(32.17 \pm 5.80)$ and the difference was statistically significant $(\mathrm{t}=11.23, \mathrm{P}<0.001)$.

Immunohistochemical staining for VEGF-A and VEGF-B in the fibrovascular proliferative membrane. As presented in 
Table IV. Optical density value of immunostain for each factor in the proliferative membrane.

\begin{tabular}{lcccr}
\hline Protein & Surgery group $(\mathrm{n}=30)$ & Drug group $(\mathrm{n}=30)$ & $\mathrm{t}$ value & P-value \\
\hline VEGF-A & $0.023 \pm 0.008$ & $0.005 \pm 0.003$ & 7.51 & $<0.001$ \\
VEGF-B & $0.009 \pm 0.005$ & $0.001 \pm 0.003$ & 11.54 & $<0.001$ \\
\hline
\end{tabular}

Values are expressed as the mean \pm standard deviation.
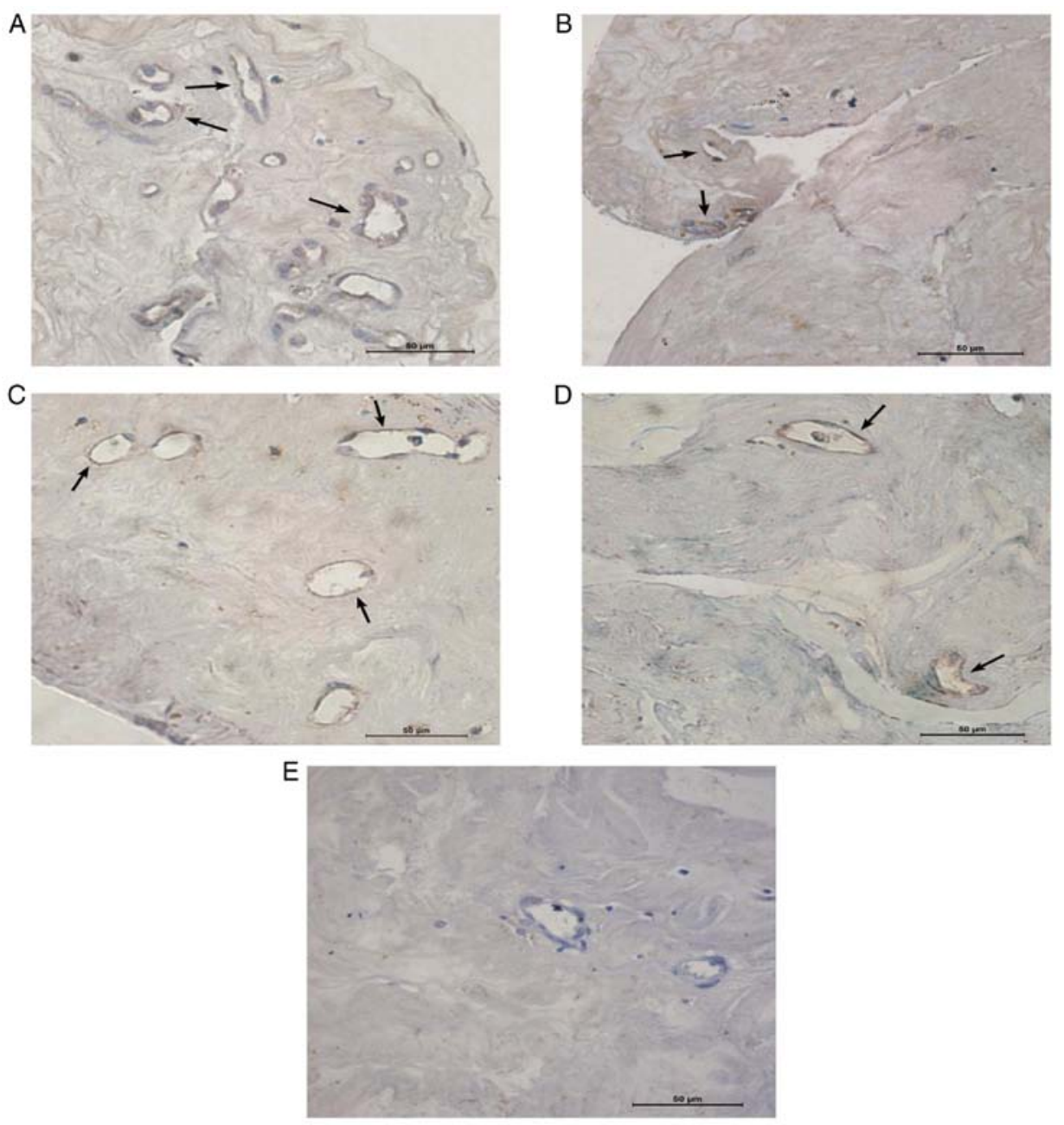

Figure 5. Immunohistochemical staining of the retinal vascular proliferative membrane (A and B) VEGF-A expression in (A) the surgery group and (B) the drug pretreatment group. (C and D) VEGF-B expression in (C) the surgery group and (D) the drug pretreatment group. (E) Negative control group (scale bars, $50 \mu \mathrm{m})$. The drug group also received surgery.

Fig. 5 and Table IV, the levels of VEGF-A and VEGF-B in the neovascular proliferation membrane of the drug group were $0.005 \pm 0.003$ and $0.001 \pm 0.003$, respectively, which were significantly lower than those of the surgery group $(0.023 \pm 0.008$ and $0.009 \pm 0.005$, respectively). Of note, the difference was statistically significant $(\mathrm{P}<0.001)$.

\section{Discussion}

VEGF family members, particularly VEGF-A, have crucial roles in neovascularization and are the major promoters of pathological angiogenesis (19). VEGF family members are capable of changing vascular morphology by increasing the number of endothelial cells and stromal cells (19). VEGF levels in patients with PDR are obviously increased and are closely correlated with the severity of DR (20). Decreasing VEGF-A levels prevents VEGF-A from inducing vascular leakage and promoting neovascularization, reducing the thickness of the central fovea of the macula in patients with diabetic macular edema (21) and improving the safety of surgery (22). The results of the present study suggested that the level of VEGF-A in the aqueous humor of patients with PDR in the combined drug-treated group was significantly lower than that of patients in the surgery group.

The occurrence and development of neovascularization in patients with PDR is a clinical problem, as hemorrhage in 
neovascularization leads to vitreous opacity, retinal proliferative membrane occurrence, retinal traction detachment and impaired visual function (19). Furthermore, intraoperative bleeding affects the surgical field and prolongs the operation time (23). The present results demonstrated that intraoperative bleeding was reduced in the combined drug treatment group during the PPV operation compared with that in the simple surgery group due to the reduced number of new blood vessels in the neovascular proliferation membrane of patients. Thus, the surgical field was clear, the operation time was shortened, the safety of the operation was improved and suffering was reduced for patients, which was similar to the results of previous studies (18-22). Accordingly, it is suggested that the widespread use of conbercept in clinical practice is able to improve the safety of PPV surgery in patients with PDR.

Previously, the concentrations of VEGF-A and VEGF-B after conbercept injection have not been determined in human eyes. The present study reported that the levels of both VEGF-A and VEGF-B obviously dropped after intravitreal injection of conbercept in patients with PDR. Furthermore, the levels of both VEGF-A and VEGF-B declined in the drug group compared with those in the PPV surgical group and the differences were statistically significant. Patients with PDR in the drug group received conbercept injection and the aqueous humor of patients was extracted 7 days after treatment but prior to surgery. However, VEGF-A and VEGF-B levels in the vitreous humor of patients with PDR were not measured prior to conbercept injection due to ethical considerations and consequently, no data for comparison were obtained. Furthermore, the drug treatment time was not prolonged to identify any changes in cytokines out of safety considerations after surgery. Due to the small number of cases in the present study, it is imperative to expand the sample size in future studies to increase the reliability of the results.

The function of VEGF-B in eye diseases remains controversial and requires further study. The present study suggested that the concentration of VEGF-B in the aqueous humor of patients with PDR decreased significantly after conbercept injection. Furthermore, the VEGF-B level declined significantly in vitreous humor after conbercept injection compared with that of patients directly receiving PPV.In most tissue types, VEGF-B has a minor role in inducing angiogenesis. Usually, a lack of VEGF-B does not affect the normal development of the retinal vasculature. However, VEGF-B may promote the survival of novel blood vessels by affecting endothelial cells, pericytes, smooth muscle cells and endothelial progenitor cells (24). When the level of VEGF-B is dropped, pathological neovascularization cannot exert pathological effects after formation because the vessels cannot stably survive, which was consistent with a previous study (25). Taken together, these results suggest that VEGF-B has a supporting role in the development of pathological neovascularization. Previous studies concerning the addition of ranibizumab and VEGF-B changes have provided conflicting results $(26,27)$ and it is controversial whether VEGF-B stimulates angiogenesis $(28,29)$. Therefore, it is essential to further identify the inhibitory effect and mechanism of VEGF-B on angiogenesis and visual acuity of patients. Furthermore, it was reported that the aqueous humor levels of placenta growth factor (PIGF) were correlated with VEGF-B, and the levels of VEGF-A, VEGF-B and PIGF decreased after intravitreal conbercept injection in patients with active PDR (30). The patient samples analyzed were mainly from the Beijing region and the samples were mainly aqueous humor. However, the samples of the present study included aqueous humor and vitreous humor. In the present study, the patient samples were analyzed from 60 patients diagnosed with DR induced by type 2 diabetes at Shanxi Eye Hospital (Taiyuan, China) between January 2016 and January 2018.

Although the outlook is promising, vitreous injection of VEGF inhibitors is not without risk. Adverse effects of anti-VEGF agents include traction retinal detachment (TRD) and endophthalmitis. It was previously reported that bevacizumab may cause TRD in patients with severe PDR (31). Fortunately, the incidence of endophthalmitis is not high. On the other hand, long-term vitreous injection of VEGF inhibitors may lead to neurodegeneration of residual healthy retina and increase the risk of choroidal circulatory disorders, which has been confirmed in mouse experiments (32). Furthermore, VEGF produced by retinal pigment epithelial cells is conducive to maintaining choroidal capillaries (33), and whether anti-VEGF treatment potentially causes pathological atrophy of the diabetic retina requires further investigation. Furthermore, the grading for preoperative vitreous hemorrhage and the intraoperative bleeding grades used in this study were established by some medical organizations $(14,15)$ rather than scales postulated in small clinical studies, this is a limitation of this study. Finally, whether the reduced capillaries in the VEGF inhibitor group are reversible, and how long it takes to normalize, and whether it affects the healing after surgery, these unsolved problems will be investigated in the future.

\section{Acknowledgements}

Not applicable.

\section{Funding}

The present study was supported by the Shanxi Province Science Foundation for Youths (grant no. 201801D221014).

\section{Availability of data and materials}

The datasets used and/or analyzed during the current study are available from the corresponding author on reasonable request.

\section{Authors' contributions}

$\mathrm{XG}$ conceived and designed the experiments. $\mathrm{YZ}$ and $\mathrm{ZG}$ performed the experiments. XZ and ZY analyzed the data. TM and GL contributed reagents/materials/analytical tools. YZ wrote the manuscript. All authors read and approved the final version of the manuscript. YZ and XG checked and confirmed the authenticity of data.

\section{Ethics approval and consent to participate}

The present study was approved by the Research Ethics Board of Shanxi Eye Hospital (Taiyuan, China; approval no. 2019B01). 


\section{Patient consent for publication}

Not applicable.

\section{Competing interests}

The authors declare that they have no competing interests.

\section{References}

1. Dehdashtian E, Mehrzadi S, Yousefi B, Hosseinzadeh A, Reiter RJ, Safa M, Ghaznavi H and Naseripour M: Diabetic retinopathy pathogenesis and the ameliorating effects of melatonin; involvement of autophagy, inflammation and oxidative stress. Life Sci 193: 20-33, 2018.

2. Yau JW, Rogers SL, Kawasaki R, Lamoureux EL, Kowalski JW, Bek T, Chen SJ, Dekker JM, Fletcher A, Grauslund J, et al: Global prevalence and major risk factors of diabetic retinopathy. Diabetes Care 35: 556-564, 2012.

3. Wilkinson CP, Ferris FL III, Klein RE, Lee PP, Agardh CD, Davis M, Dills D, Kampik A, Pararajasegaram R, Verdaguer JT, et al: Proposed international clinical diabetic retinopathy and diabetic macular edema disease severity scales. Ophthalmology 110: 1677-1682, 2003.

4. Hershberger VS, Augsburger JJ, Hutchins RK, Raymond LA and Krug S: Fibrovascular ingrowth at sclerotomy sites in vitrectomized diabetic eyes with recurrent vitreous hemorrhage: Ultrasound biomicroscopy findings. Ophthalmology 111: 1215-1221, 2004

5. Lang GE: Diabetic macular edema. Ophthalmologica 227 (Suppl 1): S21-S29, 2012

6. Waisbourd M, Goldstein M and Loewenstein A: Treatment of diabetic retinopathy with anti-VEGF drugs. Acta Ophthalmol 89 203-207, 2011

7. Nicholson BP and Schachat AP: A review of clinical trials of anti-VEGF agents for diabetic retinopathy. Graefes Arch Clin Exp Ophthalmol 248: 915-930, 2010.

8. Brown DM, Schmidt-Erfurth U, Do DV, Holz FG, Boyer DS, Midena E, Heier JS, Terasaki H, Kaiser PK, Marcus DM, et al: Intravitreal aflibercept for diabetic macular edema: 100-week results from the VISTA and VIVID studies. Ophthalmology 122 2044-2052, 2015.

9. Ciulla TA, Amador AG and Zinman B: Diabetic retinopathy and diabetic macular edema: Pathophysiology, screening, and novel therapies. Diabetes Care 26: 2653-2664, 2003.

10. Grauslund J, Green A and Sjolie AK: Prevalence and 25 year incidence of proliferative retinopathy among Danish type 1 diabetic patients. Diabetologia 52: 1829-1835, 2009

11. Yang $\mathrm{X}, \mathrm{Xu} \mathrm{J}$, Wang R, Mei Y, Lei H, Liu J, Zhang T and Zhao H: A randomized controlled trial of conbercept pretreatment before vitrectomy in proliferative diabetic retinopathy. J Ophthalmol 2016: 2473234, 2016.

12. Tetikoglu M, Yuksel Z, Aktas S, Sagdik HM and Ozcura F. VEGF-A gene polymorphisms and responses to intravitreal ranibizumab treatment in patients with diabetic macular edema Int Ophthalmol 38: 2381-2388, 2018.

13. Mesquita J, Castro de Sousa J, Vaz-Pereira S, Neves A, Tavares-Ratado P, M Santos F, A Passarinha L and T Tomaz C: VEGF-B levels in the vitreous of diabetic and non-diabetic patients with ocular diseases and its correlation with structural parameters. Med Sci (Basel) 5: 17, 2017.

14. Ahn J, Woo SJ, Chung H and Park KH: The effect of adjunctive intravitreal bevacizumab for preventing postvitrectomy hemorrhage in proliferative diabetic retinopathy. Ophthalmology 118: 2218-2226, 2011

15. Yeh PT, Yang CM, Lin YC, Chen MS and Yang CH: Bevacizumab pretreatment in vitrectomy with silicone oil for severe diabetic retinopathy. Retina 29: 768-774, 2009.

16. Pan GD, Yang JQ, Yan LN, Chu GP, Liu Q, Xiao Y and Yuan L: Reversal of multi-drug resistance by pSUPER-shRNA-mdr1 in vivo and in vitro. World J Gastroenterol 15: 431-440, 2009.
17. Takahashi S: Vascular endothelial growth factor (VEGF), VEGF receptors and their inhibitors for antiangiogenic tumor therapy. Biol Pharm Bull 34: 1785-1788, 2011.

18. Yang CM, Yeh PT, Yang $\mathrm{CH}$ and Chen MS: Bevacizumab pretreatment and long-acting gas infusion on vitreous clear-up after diabetic vitrectomy. Am J Ophthalmol 146: 211-217, 2008.

19. Folkman J: Angiogenesis: An organizing principle for drug discovery? Nat Rev Drug Discov 6: 273-286, 2007.

20. Tolentino MJ, Miller JW, Gragoudas ES, Chatzistefanou K, Ferrara N and Adamis AP: Vascular endothelial growth factor is sufficient to produce iris neovascularization and neovascular glaucoma in a nonhuman primate. Arch Ophthalmol 114: 964-970, 1996.

21. Ferrone PJ and Jonisch J: Comparison of ranibizumab $0.5 \mathrm{mg}$ versus $1.0 \mathrm{mg}$ for the treatment of patients with clinically significant diabetic macular edema: A randomized, clinical trial. Ophthalmic Surg Lasers Imaging Retina 47: 536-543, 2016.

22. Dong F, Yu C, Ding H, Shen L and Lou D: Evaluation of intravitreal ranibizumab on the surgical outcome for diabetic retinopathy with tractional retinal detachment. Medicine (Baltimore) 95: e2731, 2016.

23. Arevalo JF, Lasave AF, Kozak I, Al Rashaed S, Al Kahtani E, Maia M, Farah ME, Cutolo C, Brito M, Osorio C, et al: Preoperative bevacizumab for tractional retinal detachment in proliferative diabetic retinopathy: A prospective randomized clinical trial. Am J Ophthalmol 207: 279-287, 2019.

24. Zhang F, Tang Z, Hou X, Lennartsson J, Li Y, Koch AW, Scotney P, Lee C, Arjunan P, Dong L, et al: VEGF-B is dispensable for blood vessel growth but critical for their survival, and VEGF-B targeting inhibits pathological angiogenesis. Proc Natl Acad Sci USA 106: 6152-6157, 2009.

25. Zhong X, Huang H, Shen J, Zacchigna S, Zentilin L, Giacca M and Vinores SA: Vascular endothelial growth factor-B gene transfer exacerbates retinal and choroidal neovascularization and vasopermeability without promoting inflammation. Mol Vis 17: 492-507, 2011.

26. Puddu A, Sanguineti R, Traverso CE, Viviani GL and Nicolo M: Response to anti-VEGF-A treatment of retinal pigment epithelial cells in vitro. Eur J Ophthalmol 26: 425-430, 2016.

27. Puddu A, Sanguineti R, Traverso CE, Viviani GL and Nicolo M: Response to anti-VEGF-A treatment of endothelial cells in vitro. Exp Eye Res 146: 128-136, 2016.

28. Wafai R, Tudor EM, Angus JA and Wright CE: Vascular effects of FGF-2 and VEGF-B in rabbits with bilateral hind limb ischemia. J Vasc Res 46: 45-54, 2009.

29. Rissanen TT, Markkanen JE, Gruchala M, Heikura T, Puranen A, Kettunen MI, Kholová I, Kauppinen RA, Achen MG Stacker SA, et al: VEGF-D is the strongest angiogenic and lymphangiogenic effector among VEGFs delivered into skeletal muscle via adenoviruses. Circ Res 92: 1098-1106, 2003

30. Zhang X, Wu J, Wu C, Bian AL, Geng S and Dai RP: Comparison of aqueous humor levels of PIGF and VEGF in proliferative diabetic retinopathy before and after intravitreal conbercept injection. Diabetes Res Clin Pract 162: 108083, 2020.

31. Torres-Soriano ME, Reyna-Castelan E, Hernández-Rojas M, Garcia-Aguirre G, Kon-Jara V, Diaz-Rubio JL, Guerrero-Naranjo JL, Jimenez-Sierra JM and Quiroz-Mercado H: Tractional retinal detachment after intravitreal injection of bevacizumab in proliferative diabetic retinopathy. Retin Cases Brief Rep 3: 70-73, 2009.

32. Simo R, Sundstrom JM and Antonetti DA: Ocular Anti-VEGF therapy for diabetic retinopathy: The role of VEGF in the pathogenesis of diabetic retinopathy. Diabetes Care 37: 893-899, 2014.

33. Spilsbury K, Garrett KL, Shen WY, Constable IJ and Rakoczy PE: Overexpression of vascular endothelial growth factor (VEGF) in the retinal pigment epithelium leads to the development of choroidal neovascularization. Am J Pathol 157: 135-144, 2000.

This work is licensed under a Creative Commons Attribution-NonCommercial-NoDerivatives 4.0 International (CC BY-NC-ND 4.0) License. 